\title{
PROPERTY C, REFINABLE MAPS AND DIMENSION RAISING MAPS
}

\author{
DENNIS J. GARITY AND DALE M. ROHM
}

\begin{abstract}
We show that refinable maps defined on compacta preserve Property C. $H$. Kato has proved the analogous result for weakly infinite dimensional spaces. We also show that if $f$ is a map from a compact $C$ space $X$ onto a non $C$ space $Y$, then the set of points in $Y$ with an uncountable number of preimages is a space that does not have Property $\mathrm{C}$.
\end{abstract}

1. Introduction. Property $\mathrm{C}$ is a covering property introduced by Haver [H1, H2] and further investigated by Addis and Gresham [AG, Gr]. Every countable dimensional space is a $C$ space and each infinite dimensional $C$ space is weakly infinite dimensional. It is unknown whether Property $\mathrm{C}$ is equivalent to weak infinite dimensionality. However, R. Pol's example [Po] shows that Property C is not equivalent to countable dimensionality.

This example and results of F. D. Ancel [A1, A2] have focused renewed interest on $C$ spaces. Ancel shows that any cell-like dimension raising map defined on an ANR must have a non $C$ space as its image. A better understanding of the relationship between Property $\mathrm{C}$ and weak infinite dimensionality should lead to a better understanding of potential images of cell-like dimension raising maps.

Refinable maps have been investigated in [FR, FK, K1, K2, Pa, Ko1 and Ko2]. H. Kato shows that refinable maps defined on compacta preserve weak infinite dimensionality [K3]. A. Koyama in [Ko2] asks whether refinable maps on compacta preserve Property $\mathrm{C}$ and adds in proof a statement that they do.

The main result presented here, Theorem 1, shows that refinable maps on compacta preserve Property C. K. Sakai in a personal communication has shown that refinable maps are approximately invertible. This result combined with Ancel's work in [A2] then provides another proof of Theorem 1.

In [Ga], it is shown that a map $f$ from a $\sigma$ compact $C$ space $X$ onto a non $C$ space $Y$ has the property that $Y^{*}$ is infinite dimensional. Here $Y^{*}$ is $\left\{y \in Y \mid f^{-1}(y)\right.$ has cardinality $\geqslant c$ \}. This parallels results for weakly infinite dimensional spaces presented in [SK and Va]. In [Le], Leibo proves the following result. If $f$ is a map from a compact weakly infinite dimensional space $X$ onto a strongly infinite

Received by the editors September 9, 1985 and, in revised form, October 14, 1985. Presented at the 92nd Annual Meeting of the AMS in New Orleans, Louisiana on January 10, 1986.

1980 Mathematics Subject Classification (1985 Revision). Primary 54F45, 54C10.

Key words and phrases. Property C, refinable map, countable dimensional, weakly infinite dimensional. 
dimensional space $Y$, then $Y^{*}$ is strongly infinite dimensional. In Theorem 2, we show that the analogous result holds for spaces with Property $C$.

Finally, we would like to thank Doug Curtis for pointing out additional references and suggesting improvements in the paper.

2. Definitions. All spaces will be separable metric. A space $X$ has Property $\mathrm{C}$ or is a $C$ space if for each sequence $\mathscr{U}_{1}, \mathscr{U}_{2}, \ldots$ of open covers of $X$, there exists a sequence $\mathscr{V}_{1}, \mathscr{V}_{2}, \ldots$ satisfying the following conditions:

1. Each $\mathscr{V}_{i}$ is a pairwise disjoint collection of open sets.

2. Each $V$ in $\mathscr{V}_{i}$ is contained in some $U$ in $\mathscr{U}_{i}$.

3. $\cup \mathscr{V}_{i}$ is a cover of $X$.

Such a sequence is called a $C$ refinement of the sequence of covers $\left(\mathscr{U}_{i}\right)$.

A map between spaces is a continuous function. An $A N R$ is an absolute neighborhood retract for the class of metric spaces. A map $f: X \rightarrow Y$ between compacta is an $\varepsilon$ map for some $\varepsilon>0$ if, for each $y \in Y$, diam $\left(f^{-1}(y)\right)<\varepsilon$. A map $r: X \rightarrow Y$ between compacta is refinable if for each $\varepsilon>0$ there exists a surjective $\varepsilon$ map, called an $\varepsilon$ refinement of $r, r_{\varepsilon}: X \rightarrow Y$, so that $d\left(r, r_{\varepsilon}\right)<\varepsilon$.

If $\left\{A_{i}\right\}, 1 \leqslant i<\infty$, is a sequence of closed sets in a compactum $X$, the $\lim \sup _{i} A_{i}$ is $\left\{x \in X \mid\right.$ for each open set $U$ containing $x, U$ intersects infinitely many of the $\left.A_{i}\right\}$. Equivalently, $\lim \sup _{i} A_{i}=\left\{x \in X \mid\right.$ there exists $x_{i_{k}} \in A_{i_{k}}$ with $\left.\lim _{k \rightarrow \infty} x_{i_{k}}=x\right\}$. Note that if $U$ is an open set in a compactum $X$ so that $\lim _{\sup _{i}} A_{i} \subset U$, then there exists an $N$ so that $A_{n} \subset U$ for each $n>N$.

If $f: X \rightarrow Y$ is a map, $Y^{*}$ is defined to be $\left\{y \in Y \mid f^{-1}(y)\right.$ has cardinality $\left.\geqslant c\right\}$. Here $c$ is the cardinality of $\mathbf{R}$.

3. Refinable maps. In proving Theorem 1 , we need the following result due to Loncar and Mardesic.

LEMMA 1 [LM]. Let $f: X \rightarrow A$ be a map from a compactum $X$ to an ANR A. Given any $\varepsilon>0$, there exists $a \delta>0$ so that for any surjective $\delta$ map $g: X \rightarrow Y$ there exists a map $h: Y \rightarrow A$ with $d(f, h g)<\varepsilon$.

THEOREM 1. Let $r: X \rightarrow Y$ be a surjective refinable map between compacta. If $X$ has Property $\mathrm{C}$, then $Y$ has Property $\mathrm{C}$.

Proof. For each $i \in \mathbf{N}$ let $\mathscr{U}_{i}$ be an open cover of $Y$. Since $Y$ is compact, we may assume each $\mathscr{U}_{i}=\left\{U_{i j}\right\}, 1 \leqslant j \leqslant N_{i}$. For each $i \in \mathbf{N}$ let $r^{-1}\left(\mathscr{U}_{i}\right)=\left\{r^{-1}(U) \mid U \in\right.$ $\left.\mathscr{U}_{i}\right\}$. Since $X$ is compact and has Property $\mathrm{C}$, there exists an integer $N$ and collections of open sets $\mathscr{V}_{i}$ of $X, 1 \leqslant i \leqslant N$, so that

1. For each $i, 1 \leqslant i \leqslant N, \mathscr{V}_{i}=\left\{\mathscr{V}_{i j}\right\}, 1 \leqslant j \leqslant N_{i}$, is a collection of open sets with pairwise disjoint closures.

2. For each $i, 1 \leqslant i \leqslant N$, and for each $j, 1 \leqslant j \leqslant N_{i}, V_{i j} \subset \bar{V}_{i j} \subset r^{-1}\left(U_{i j}\right)$.

3. $\bigcup_{i=1}^{N} \mathscr{V}_{i}$ is an open cover of $X$.

Now for each $i=1, \ldots, N$ let $A_{i}$ denote the join of $N_{i}$ intervals, joined at a common endpoint. Label the remaining endpoints $a_{1}, a_{2}, \ldots, a_{N_{i}}$. To avoid ambiguity, an endpoint $a_{j}$ will always be referred to together with the $A_{i}$ of which it is an endpoint. The $A_{i}$ are of course ANRs. 
For each $i=1, \ldots, N$, we can choose maps $f_{i}: X \rightarrow A_{i}$ so that, for each $j=1, \ldots, N_{i}$,

$$
f_{i}^{-1}\left(a_{j}\right)=r^{-1}\left(Y \backslash \bigcup_{k \neq j} U_{i k}\right) \cup \bar{V}_{i j} .
$$

Since $f_{i}^{-1}\left(a_{j}\right) \subset r^{-1}\left(U_{i j}\right)$ we see that $f_{i}\left(r^{-1}\left(Y \backslash U_{i j}\right)\right)$ is a closed set in $A$; disjoint from the endpoint $a_{j}$.

Using complete normality, for each $i, 1 \leqslant i \leqslant N$, we can choose open sets $M_{i j}$ and $N_{i j}, 1 \leqslant j \leqslant N_{i}$, in $A_{i}$ so that

1. $a_{j} \in N_{i j}$.

2. For each $i,\left\{N_{i j} \mid i \leqslant j \leqslant N_{i}\right\}$ is a pairwise disjoint collection.

3. $f_{i}\left(r^{-1}\left(Y \backslash U_{i j}\right)\right) \subset M_{i j}$.

4. $d\left(\overline{M_{i j}}, \overline{N_{i j}}\right) \equiv D_{i j}>0$.

Fix $k \in \mathbf{N}$ and $i \in\{1, \ldots, N\}$. For each such $i$ and $k$, the lemma guarantees the existence of a number $\delta_{i k}>0$ so that if $g: X \rightarrow Y$ is any surjective $\delta_{i k}$ map then there exists a map $h_{i k}: Y \rightarrow A_{i}$ with $d\left(f_{i}, h_{i k} \circ g\right)<1 / k$. In particular, if we let $\delta_{k}=\min \left\{1 / k, \delta_{k}\right\}, 1 \leqslant i \leqslant N$, then the refinability of $r$ allows us to choose a map $r_{k}: X \rightarrow Y$ so that $r_{k}$ is a $\delta_{k}$ refinement of $r$. In addition, since $r_{k}$ is then a $\delta_{i k}$ map for each $i, 1 \leqslant i \leqslant N$, Lemma 1 can be applied to choose maps $h_{i k}: Y \rightarrow A_{i}$ with $d\left(f_{i}, h_{i k} \circ r_{k}\right)<1 / k$.

We thus obtain two sequences of maps. We obtain a sequence $\left(r_{k}\right)$ of maps from $X$ onto $Y$ where $r_{k}$ is a $1 / k$ refinement of $r$. We also obtain for each $i, 1 \leqslant i \leqslant N$, a sequence $\left(h_{i k}\right)$ of maps from $Y$ to $A_{i}$ so that $d\left(f_{i}, h_{i k} \circ r_{k}\right)<1 / k$.

ClaIM. $\lim \sup _{k}\left(r_{k}^{-1}\left(Y \backslash U_{i j}\right)\right) \subset r^{-1}\left(Y \backslash U_{i j}\right) \subset f_{i}^{-1}\left(M_{i j}\right)$.

For, let $x \in \lim \sup _{k} r_{k}^{-1}\left(Y \backslash U_{i j}\right)$. Then there exists a sequence $x_{k_{l}} \in r_{k_{l}}\left(Y \backslash U_{i j}\right)$ so that $x_{k_{l}} \rightarrow x$ as $l \rightarrow \infty$. Now,

$$
d\left(r(x), Y \backslash U_{i j}\right) \leqslant d\left(r_{k_{l}}\left(x_{k_{l}}\right), Y \backslash U_{i j}\right)+d\left(r_{k_{l}}\left(x_{k_{l}}\right), r\left(x_{k_{l}}\right)\right)+d\left(r\left(x_{k_{l}}\right), r(x)\right) .
$$

This goes to 0 as $l \rightarrow \infty$. Since $Y \backslash U_{i j}$ is closed, this completes the proof of the claim.

It is now possible to choose $m$ large enough so that for each $i, 1 \leqslant i \leqslant N$, and for each $j, 1 \leqslant j \leqslant N_{i}$,

$$
m^{-1}<d\left(a_{j}, A_{i} \backslash N_{i j}\right), \quad m^{-1}<D_{i j} \quad \text { and } \quad r_{m}^{-1}\left(Y \backslash U_{i j}\right) \subset f_{i}^{-1}\left(M_{i j}\right) .
$$

Finally, let $W_{i j}=h_{i m}^{-1}\left(N_{i j}\right)$ and $\mathscr{W}_{i}=\left\{W_{i j} \mid 1 \leqslant j \leqslant N_{i}\right\}$.

For any fixed $i,\left\{N_{i j} \mid 1 \leqslant j \leqslant N_{i}\right\}$ is a collection of pairwise disjoint open sets in $A_{i}$. Thus each $\mathscr{W}_{i}$ is also a collection of pairwise disjoint open sets in $Y$. To show that $Y$ has Property $\mathrm{C}$, it only remains to show that $\mathscr{W}_{i}$ refines $\mathscr{U}_{i}$ for each $i$, and that $\cup_{i=1}^{N} \mathscr{W}_{i}$ covers $Y$.

Suppose $y \in W_{i j}$ and that $y \in Y \backslash U_{i j}$. Let $x \in r_{m}^{-1}(y)$. By our choice of $m$, $x \in f_{i}^{-1}\left(M_{i j}\right)$, so that $f_{i}(x) \in M_{i j}$. Also, $h_{i m}(y)=h_{i m} \circ r_{m}(x) \in N_{i j}$, but then $d\left(f_{i}(x), h_{i m} \circ r_{m}(x)\right) \geqslant d\left(\overline{M_{i j}}, \overline{N_{i j}}\right)=D_{i j}>1 / m$, which is a contradiction. Thus $\mathscr{W}_{i}$ refines $\mathscr{U}_{i}$. 
Next, let $y \in Y$ and $x \in r_{m}^{-1}(y)$. Then there exists $i$ and $j$ so that $x \in V_{i j}$. Now

$$
\begin{aligned}
d\left(f_{i}(x), h_{i m} \circ r_{m}(x)\right) & =d\left(a_{j}, h_{i m}(y)\right)<1 / m \\
& <d\left(a_{j}, A_{i} \backslash N_{i j}\right) .
\end{aligned}
$$

Since $a_{j} \leqslant N_{i j} \subset A_{i}$, we have $h_{i m}(y) \leqslant N_{i j}$. Thus $y \leqslant h_{i m}^{-1}\left(N_{i j}\right)=W_{i j}$ and $\bigcup_{i=1}^{N} \mathscr{W}_{i}$ covers $Y$. So $Y$ has Property $C$.

In [Ro], D. Rohm shows that weak infinite dimensonality can be defined using a covering property similar to that used in defining Property $\mathrm{C}$. The difference is that binary open covers rather than arbitrary open covers are used. Using this definition, the above proof provides an alternate proof of Kato's Theorem that refinable maps preserve weak infinite dimensionality [K3].

4. Dimension raising maps. If Property $\mathrm{C}$ is interpreted as a dimension like property, Theorem 1 can be read to say that refinable maps on $C$ spaces do not raise dimension. Theorem 2 explains properties of closed dimension raising maps defined on $C$ spaces.

Lemma 2. If $X$ is a non $C$ space and $A$ is a subspace of $X$ that has Property $\mathrm{C}$, then there exists a subspace $F$ of $X \backslash A$ that is a non $C$ space and that is closed in $X$.

Proof. Let $\mathscr{U}_{i}, 1 \leqslant i<\infty$, be a sequence of open covers of $X$ that has no $C$ refinement. Then the sequence of covers $\mathscr{U}_{2 i}, 1 \leqslant i<\infty$, restricted to $A$ does have a $C$ refinement $\mathscr{V}_{2 i}, 1 \leqslant i<\infty$. We may assume that the sets $V$ in $\mathscr{V}_{2 i}$ are actually pairwise disjoint and open in $X$. Let $V=\bigcup_{i=1}^{\infty}\left(\cup \mathscr{V}_{2 i}\right)$. Then $\mathscr{V}$ is an open subset of $X$ that contains $A$. Let $F=X \backslash \mathscr{V}$. Then the sequence of covers $\mathscr{U}_{2 i+1}, 0 \leqslant i<\infty$, restricted to $F$ cannot have a $C$ refinement. Otherwise, the sequence of covers $\mathscr{U}$, $1 \leqslant i<\infty$, of $X$ would have a $C$ refinement. So $F$ is a non $C$ space.

THEOREM 2. Let $f$ be a closed map from a $\sigma$ compact $C$ space $X$ onto a non $C$ space $Y$. Then $Y^{*}$ does not have Property $\mathrm{C}$.

Proof. By [Ga] $Y^{*}$ is nonempty. Assume that $Y^{*}$ had Property $\mathrm{C}$. The lemma then implies that there is a non $C$ subspace $F$ of $Y \backslash Y^{*}$ so that $F$ is closed in $Y$. Let $Z=f^{-1}(F)$. Then $f \mid Z: Z \rightarrow F$ is a map satisfying the hypotheses of the theorem. So by [Ga], there exists a $y \in F$ so that $f^{-1}(y)$ has cardinality $\geqslant c$. This contradicts the fact that $y \in F \subset Y \backslash Y^{*}$.

\section{REFERENCES}

[AG] D. F. Addis and J. H. Gresham, A class of infinite-dimensional spaces. Part I: Dimension theory and Alexandroff's problem, Fund. Math. 101 (1978), 195-205.

[A1] F. D. Ancel, The role of countable dimensionality in the theory of cell-like relations, Trans. Amer. Math. Soc. 287 (1985), 1-40.

[A2] F. D. Ancel, Proper hereditary shape equivalences preserve property C, Topology Appl. 19 (1985), 71-74.

[FK] J. Ford and G. Kozlowski, Refinable maps on ANR, Topology Appl. 11 (1980), 247-263.

[FR] J. Ford and J. W. Rogers, Jr., Refinable maps, Colloq. Math. 39 (1978), 263-269.

[Ga] D. J. Garity, Property C and closed maps, Topology Appl. (to appear).

[Gr] J. H. Gresham, A class of infinite-dimensional spaces. Part II: An extension theorem and the theory of retracts, Fund. Math. CVI (1980), 237-245. 
[H1] W. E. Haver, A covering property for metric spaces, Topology, Conf., Virginia Polytechnic Institute and State University (R. E. Diockman and P. Fletcher, editors), Lecture Notes in Math., vol. 375, Springer-Verlag, New York, 1974, pp. 108-113.

[H2] _ _ A near selection theorem, General Topology Appl. 9 (1978), 117-124.

[K1] H. Kato, Refinable maps onto locally n-connected compacta, Tsukuba J. Math. 4 (1980), 83-88.

[K2] _ Refinable maps in the theory of shape, Fund. Math. 113 (1981), 119-129.

[K3] _ A note on infinite-dimension under refinable maps, Proc. Amer. Math. Soc. 88 (1983), $177-180$.

[Ko1] A. Koyama, Refinable maps in dimension theory, Topology Appl. 17 (1984), 247-255.

[Ko2] A. Koyama," Some topics in dimension theory, questions and answers, Topology 1 (1983), 100-106.

[Le] I. M. Leibo, On closed mappings of infinite dimensional spaces, Soviet Math. Dokl. 12 (1971), 1111-1113 = Dokl. Akad. Nauk SSSR 199 (1971), No. 3.

[LM] I. Loncar and S. Mardesic, A note on inverse sequences of ANR's, Glasnik Mat. 3 (23) (1968), 41-48.

[Pa] P. R. Patten, Refinable maps and generalized absolute neighborhood retracts, Topology Appl. 14 (1982), 183-188.

[Po] R. Pol, A weakly infinite dimensional compactum which is not countable dimensional, Proc. Amer. Math. Soc. 82 (1981), 634-636.

[Ro] D. Röhm, Hereditary shape equivalences preserve weak infinite dimensionality, preprint.

[Sk] E. G. Sklyarenko, Two theorems on infinite dimensional spaces, Dokl. Akad. Nauk SSSR 143 (1962), 1053-1056 = Soviet Math. Dokl. 3 (1962), 547-550.

[Va] A. I. Vainstein, $A$ class of infinite-dimensional spaces, Mat. Sb. 79 (121) (1969) $=$ Math. USSR-Sb. 8 (1969), 409-417.

Department of Mathematics, Oregon State University, Corvallis, Oregon 97331 